\title{
Hyers-ulam stability of exact second-order linear differential equations
}

\author{
Mohammad Bagher Ghaemi ${ }^{1}$, Madjid Eshaghi Gordji ${ }^{2}$, Badrkhan Alizadeh ${ }^{3}$ and Choonkil Park ${ }^{4 *}$
}

\author{
* Correspondence: baak@hanyang. \\ ac.kr \\ ${ }^{4}$ Department of Mathematics, \\ Research Institute for Natural \\ Sciences, Hanyang University, Seoul \\ 133-791, South Korea \\ Full list of author information is \\ available at the end of the article
}

\begin{abstract}
In this article, we prove the Hyers-Ulam stability of exact second-order linear differential equations. As a consequence, we show the Hyers-Ulam stability of the following equations: second-order linear differential equation with constant coefficients, Euler differential equation, Hermite's differential equation, Cheybyshev's differential equation, and Legendre's differential equation. The result generalizes the main results of Jung and Min, and Li and Shen.
\end{abstract}

Mathematics Subject Classification (2010): 26D10; 34K20; 39B52; 39B82; $46 B 99$.

Keywords: Hyers-Ulam stability, exact second-order linear differential equation

\section{Introduction}

The stability of functional equations was first introduced by Ulam [1]. Hyers [2] gave a partial solution of Ulam's problem for the case of approximate additive mappings in the context of Banach spaces. Rassias [3] generalized the theorem of Hyers by considering the stability problem with unbounded Cauchy differences $\|f(x+y)-f(x)-f(y)\|$ $\leq \varepsilon\left(\|x\|^{p}+\|y\|^{p}\right)(\varepsilon>0, p \in[0,1))$.

Let $X$ be a normed space over a scalar field $\mathbb{K}$ and let $I$ be an open interval. Assume that for any function $f: I \rightarrow X(y=f(x))$ satisfying the differential inequality

$$
\left\|a_{n}(t) y^{(n)}(t)+a_{n-1}(t) y^{(n-1)}(t)+\cdots+a_{1}(t) y^{\prime}(t)+a_{0}(t) y(t)+h(t)\right\| \leq \varepsilon
$$

for all $t \in I$ and some $\varepsilon \geq 0$, there exists a function $f_{0}: I \rightarrow X\left(y=f_{0}(x)\right)$ satisfying

$$
a_{n}(t) y^{(n)}(t)+a_{n-1}(t) y^{(n-1)}(t)+\cdots+a_{1}(t) y^{\prime}(t)+a_{0}(t) y(t)+h(t)=0 .
$$

and $\left\|f(t)-f_{0}(t)\right\| \leq K(\varepsilon)$ for all $t \in I$. Here $\lim _{\varepsilon \rightarrow 0} K(\varepsilon)=0$. Then we say that the above differential equation has the Hyers-Ulam stability.

If the above statement is also true when we replace $\varepsilon$ and $K(\varepsilon)$ by $\phi(t)$ and $\varphi(t)$, where $\phi, \varphi: I \rightarrow[0, \infty)$ are functions not depending on $f$ and $f_{0}$ explicitly, then we say that the corresponding differential equation has the Hyers-Ulam stability.

The Hyers-Ulam stability of the differential equation $y^{\prime}=y$ was first investigated by Alsina and Ger [4]. This result has been generalized by Takahasi et al. [5] for the Banach space-valued differential equation $y^{\prime}=\lambda y$. Jung [6] proved the Hyers-Ulam stability of a linear differential equation of first-order.

Theorem 1.1. ([6]) Let $y: I \rightarrow \mathbb{R}$ be a continuously differentiable function satisfying the differential inequality

(C) 2012 Ghaemi et al; licensee Springer. This is an Open Access article distributed under the terms of the Creative Commons Attribution License (http://creativecommons.org/licenses/by/2.0), which permits unrestricted use, distribution, and reproduction in any medium, provided the original work is properly cited. 


$$
\left|\gamma^{\prime}(t)+g(t) y(t)+h(t)\right| \leq \varphi(t)
$$

for all $t \in I$, where $g, h: I \rightarrow \mathbb{R}$ are continuous functions and $\phi: I \rightarrow[0, \infty)$ is a function. Assume that

(a) $g(t)$ and $\exp \left\{\int_{a}^{t} g(u) d u\right\} h(t)$ are integrable on $(a, c)$ for each $c \in I$;

(b) $\varphi(t) \exp \left\{\int_{a}^{t} g(u) d u\right\}$ is integrable on $I$.

Then there exists a unique real number $x$ such that

$$
\begin{gathered}
\left|y(t)-\exp \left\{-\int_{a}^{t} g(u) d u\right\}\left[x-\int_{a}^{t} \exp \left\{\int_{a}^{v} g(u) d u\right\} h(v) d v\right]\right| \\
\leq\left|\exp \left\{-\left(\int_{a}^{t} g(u) d u\right)\right\} \int_{t}^{b} \varphi(v) \exp \left\{\left(\int_{a}^{v} g(u) d u\right)\right\} d v\right|
\end{gathered}
$$

for all $t \in I$.

In this article, we prove the Hyers-Ulam stability of exact second-order linear differential equations (see [7]). A general second-order differential equation is of the form

$$
p_{0}(x) y^{\prime \prime}+p_{1}(x) y^{\prime}+p_{2}(x) y+f(x)=0
$$

and it is exact if

$$
p_{0}^{\prime \prime}(x)-p_{1}^{\prime}(x)+p_{2}(x)=0 .
$$

\section{Main results}

In this section, let $I=(a, b)$ be an open interval with $-\infty \leq a<b \leq \infty$. In the following theorem, $p(x)=\left(p_{0}(x)\right)^{-1}\left(p_{1}(x)-p_{0}^{\prime}(x)\right)$ and $k=-\left[p_{0}(a) \gamma^{\prime}(a)-p_{0}^{\prime}(a) y(a)+p_{1}(a) \gamma(a)\right]$. Taking some idea from [6], we investigate the Hyers-Ulam stability of exact secondorder linear differential equations. For the sake of convenience, we assume that all the integrals and derivations exist.

Theorem 2.1. Let $p_{0}, p_{1}, p_{2}, f: I \rightarrow \mathbb{R} b$ e continuous functions with $p_{0}(x) \neq 0$ for all $x$ $\in I$, and let $\phi: I \rightarrow[0, \infty)$ be a function. Assume that $y: I \rightarrow \mathbb{R}$ is a twice continuously differentiable function satisfying the differential inequality

$$
\left|p_{0}(x) y^{\prime \prime}+p_{1}(x) y^{\prime}+p_{2}(x) y+f(x)\right| \leq \varphi(x)
$$

for all $x \in I$ and (2) is true. Then there exists a solution $y_{0}: I \rightarrow \mathbb{R} o f(1)$ such that

$$
\begin{aligned}
& \left|\gamma(x)-y_{0}(x)\right| \\
& \leq \exp \left\{-\left(\int_{a}^{x} p(u) d u\right)\right\} \int_{x}^{b}\left(\left|p_{0}(v)\right|^{-1} \int_{a}^{v} \varphi(t) d t\right) \exp \left\{\left(\int_{a}^{v} p(u) d u\right)\right\} d v
\end{aligned}
$$

for all $x \in I$. 
Proof. It follows from (2) and (3) that

$$
\begin{aligned}
\mid p_{0}(x) & y^{\prime \prime}+p_{1}(x) y^{\prime}+p_{2}(x) y+f(x) \mid \\
= & \left|\left(p_{0}(x) y^{\prime}-p_{0}^{\prime}(x) y\right)^{\prime}+\left(p_{1}(x) y\right)^{\prime}+\left[p_{0}^{\prime \prime}(x)-p_{1}^{\prime}(x)+p_{2}(x)\right] y+f(x)\right| \\
= & \left|\left(p_{0}(x) y^{\prime}-p_{0}^{\prime}(x) y\right)^{\prime}+\left(p_{1}(x) y\right)^{\prime}+f(x)\right| \leq \varphi(x)
\end{aligned}
$$

So we have

$$
-\varphi(x) \leq\left(p_{0}(x) y^{\prime}-p_{0}^{\prime}(x) y\right)^{\prime}+\left(p_{1}(x) y\right)^{\prime}+f(x) \leq \varphi(x) .
$$

Integrating (4) from $a$ to $x$ for each $x \in I$, we get

$$
\begin{aligned}
& \left|p_{0}(x) y^{\prime}-p_{0}^{\prime}(x) y+p_{1}(x) y+k+\int_{a}^{x} f(t) d t\right| \\
& =\left|p_{0}(x)\right| y^{\prime}+\left(p_{0}(x)\right)^{-1}\left(p_{1}(x)-p_{o}^{\prime}(x)\right) y+\left(p_{0}(x)\right)^{-1}\left(k+\int_{a}^{x} f(t) d t\right) \mid \\
& \leq \int_{a}^{x} \varphi(t) d t .
\end{aligned}
$$

Dividing both sides of the inequality (5) by $\left|p_{0}(x)\right|$, we obtain

$$
\begin{array}{r}
\left|y^{\prime}+\left(p_{0}(x)\right)^{-1}\left(p_{1}(x)-p_{o}^{\prime}(x)\right) y+\left(p_{0}(x)\right)^{-1}\left(k+\int_{a}^{x} f(t) d t\right)\right| \\
\leq\left|p_{0}(x)\right|^{-1} \int_{a}^{x} \varphi(t) d t .
\end{array}
$$

If we set

$$
p(x)=\left(p_{0}(x)\right)^{-1}\left(p_{1}(x)-p_{0}^{\prime}(x)\right), h(x)=\left(p_{0}(x)\right)^{-1}\left(k+\int_{a}^{x} f(t) d t\right)
$$

and $\varphi_{1}(x)=\left|p_{0}(x)\right|^{-1} \int_{a}^{x} \varphi(t) d t$ in (6), then we have

$$
\left|y^{\prime}+p(x) y+h(x)\right| \leq \varphi_{1}(x)
$$

Now we are in the situation of Theorem 1.1, that is, there exists a unique $z \in \mathbb{R}$ such that

$$
\begin{aligned}
& \left|\gamma(x)-\exp \left\{-\int_{a}^{x} p(u) d u\right\}\left[z-\int_{a}^{x} \exp \left\{\int_{a}^{v} p(u) d u\right\} h(v) d v\right]\right| \\
& \leq \exp \left\{-\left(\int_{a}^{x} p(u) d u\right)\right\} \int_{x}^{b} \varphi_{1}(v) \exp \left\{\left(\int_{a}^{v} p(u) d u\right)\right\} d v \\
= & \exp \left\{-\left(\int_{a}^{x} p(u) d u\right)\right\} \int_{x}^{b}\left(\left|p_{0}(v)\right|^{-1} \int_{a}^{v} \varphi(t) d t\right) \exp \left\{\left(\int_{a}^{v} p(u) d u\right)\right\} d v
\end{aligned}
$$


for all $x \in I$.

It is easy to show that

$$
y_{0}(x)=\exp \left\{-\int_{a}^{x} p(u) d u\right\}\left[z-\int_{a}^{x} \exp \left\{\int_{a}^{v} p(u) d u\right\} h(v) d v\right]
$$

is a solution of (1) with the condition (2).

If (1) is multiplied by a function $\mu(x)$ such that the resulting equation is exact, that is,

$$
(\mu(x))\left[p_{0}(x) y^{\prime \prime}+p_{1}(x) y^{\prime}+p_{2}(x) y+f(x)\right]=0
$$

and

$$
\left(\mu(x) p_{0}(x)\right)^{\prime \prime}-\left(p_{1}(x) \mu(x)\right)^{\prime}+p_{2}(x) \mu(x)=0,
$$

then we say that $\mu(x)$ is an integrating factor of the Equation (1) (see [7]).

Corollary 2.2. Let $p_{0}, p_{1}, p_{2}, \mu: I \rightarrow \mathbb{R}$ be continuous functions with $p_{0}(x) \neq 0$ and $\mu(x) \neq 0$ for all $x \in I$, and let $\phi: I \rightarrow[0, \infty)$ be a function. Assume that $y: I \rightarrow \mathbb{R}$ is a twice continuously differentiable function satisfying the differential inequality

$$
\left|(\mu(x))\left[p_{0}(x) y^{\prime \prime}+p_{1}(x) y^{\prime}+p_{2}(x) y+f(x)\right]\right| \leq \varphi(x)
$$

for all $x \in I$ and (8) is true. Then there exists a solution $y_{0}: I \rightarrow \mathbb{R} o f(7)$ such that

$$
\begin{aligned}
& \left|y(x)-y_{0}(x)\right| \\
& \leq \exp \left\{-\left(\int_{a}^{x} p(u) d u\right)\right\} \int_{x}^{b}\left(\left|\mu(v) p_{0}(v)\right|^{-1} \int_{a}^{v} \varphi(t) d t\right) \exp \left\{\left(\int_{a}^{v} p(u) d u\right)\right\} d v
\end{aligned}
$$

for all $x \in I$, where $p(x)=\left(\mu(x) p_{0}(x)\right)^{-1}\left[\mu(x) p_{1}(x)-\left(\mu(x) p_{0}(x)\right)^{\prime}\right]$.

Proof. It follows from Theorem 2.1 that there exists a unique $z \in \mathbb{R}$ such that

$$
y_{0}(x)=\exp \left\{-\int_{a}^{x} p(u) d u\right\}\left[z-\int_{a}^{x} \exp \left\{\int_{a}^{v} p(u) d u\right\} h(v) d v\right]
$$

is a solution of (7) with the condition (8), where

$$
p(x)=\left(\mu(x) p_{0}(x)\right)^{-1}\left[\mu(x) p_{1}(x)-\left(\mu(x) p_{0}(x)\right)^{\prime}\right]
$$

and

$$
h(x)=\left(\mu(x) p_{0}(x)\right)^{-1}\left(k+\int_{a}^{x} \mu(t) f(t) d t\right)
$$

with

$$
k=-\left[\mu(a) p_{0}(a) \gamma^{\prime}(a)-\left(\mu(a) p_{0}(a)\right)^{\prime} \gamma(a)+\mu(a) p_{1}(a) y(a)\right],
$$

as desired. $\square$

1. Li and Shen [8] proved the Hyers-Ulam stability of second-order linear differential equations with constant coefficients 


$$
y^{\prime \prime}+c y^{\prime}+b y+f(x)=0
$$

where the characteristic equation $\lambda^{2}+c \lambda+b=0$ has two positive roots.

Now, it follows from (7) and (8) that $\mu(x)$ is an integrating factor for (10) if it satisfies

$$
\mu^{\prime \prime}(x)-c \mu^{\prime}(x)+b \mu(x)=0 .
$$

It is well-known that $\mu(x)=\exp (m x)$, where $m=\frac{c \pm \sqrt{c^{2}-4 b}}{2}$, is a solution of (11) and consequently, it is an integrating factor of (10). Now the following corollaries are the generalization of [[8], Theorems 2.1 and 2.2].

Corollary 2.3. Consider the Equation (10). Let $c^{2}-4 b \geq 0$, $m=\frac{c \pm \sqrt{c^{2}-4 b}}{2}, f: I \rightarrow \mathbb{R}$ be a continuous function and let $\phi: I \rightarrow[0, \infty)$ be a function. Assume that $y: I \rightarrow \mathbb{R}$ is a twice continuously differentiable function satisfying the differential inequality

$$
\left|y^{\prime \prime}+c y^{\prime}+b y+f(x)\right| \leq \varphi(x)
$$

for all $x \in I$. Then there exists a solution $y_{0}: I \rightarrow \mathbb{R}$ of $(10)$ such that

$$
\left|\gamma(x)-y_{0}(x)\right| \leq \exp \{(m-c)(x-a)\} \int_{x}^{b}\left(\exp (-m v) \int_{a}^{v} \exp (m t) \varphi(t)\right) \exp \{(c-m)(x-a)\} d v
$$

for all $x \in I$.

Proof. $\mu(x)=\exp (m x)$ is an integrating factor of (10) when $c^{2}-4 b \geq 0$ and $m=\frac{c \pm \sqrt{c^{2}-4 b}}{2}$ (the paragraph preceding of this corollary). By (12), we obtain

$$
\exp (m x)\left|y^{\prime \prime}+c y^{\prime}+b y+f(x)\right| \leq \exp (m x) \varphi(x)
$$

for all $x \in I$. Using Corollary 2.2 with $\phi_{1}(x)=\exp (m x) \phi(x)$ instead of $\phi(x)$ and with (13) instead of (9), we conclude that there exists a unique $z \in \mathbb{R}$ such that

$$
y_{0}(x)=\exp \{(m-c)(x-a)\}\left[z-\int_{a}^{x} \exp \{(c-m)(v-a)\}\left(k+\int_{a}^{v} \exp (m x) f(x) d x\right) d v\right]
$$

where $k=-\left[\exp (m a) y^{\prime}(a)-m \exp (m a) y(a)+c \exp (m a) y(a)\right]$, for all $x \in I$, is a solution of (10) and

$$
\left|\gamma(x)-y_{0}(x)\right| \leq \exp \{(m-c)(x-a)\} \int_{x}^{b}\left(\exp (m v) \int_{a}^{v} \exp (m t) \varphi(t)\right) \exp \{(c-m)(v-a)\} d v,
$$

as desired. $\square$

Corollary 2.4. Consider the Equation (10). Let $c^{2}-4 b<0$, $m=\frac{c \pm \sqrt{c^{2}-4 b}}{2}=\alpha \pm i \beta, f: I \rightarrow \mathbb{R}$ be a continuous function and let $\phi: I \rightarrow[0, \infty)$ be a function. Assume that $y: I \rightarrow \mathbb{R}$ is a twice continuously differentiable function satisfying the differential inequality 


$$
\left|y^{\prime \prime}+c y^{\prime}+b y+f(x)\right| \leq \varphi(x)
$$

for all $x \in I$. Then there exists a solution $y_{0}: I \rightarrow \mathbb{R}$ of $(10)$ such that

$$
\begin{aligned}
& \left|\gamma(x)-y_{0}(x)\right| \\
& \leq \exp \left\{-\left(\int_{a}^{x} p(u) d u\right)\right\} \int_{x}^{b}\left(|\mu(v)|^{-1} \int_{a}^{v} \exp (\alpha t) \varphi(t) d t\right) \exp \left\{\left(\int_{a}^{v} p(u) d u\right)\right\} d v
\end{aligned}
$$

for all $x \in I$, where $\mu(x)=\exp (\alpha x) \cos \beta x$ and $p(u)=[c-\alpha+\beta \tan \beta x]$.

Proof. It is easy to show that

$$
\begin{aligned}
\exp (\alpha x)\left|(\cos \beta x)\left(y^{\prime \prime}+c y^{\prime}+b y+f(x)\right)\right| & \leq \exp (\alpha x)\left|y^{\prime \prime}+a y^{\prime}+b y+f(x)\right| \\
& \leq \exp (\alpha x) \varphi(x)
\end{aligned}
$$

for all $x \in I$. Now, similar to Corollary 2.3, there exists a unique $z \in \mathbb{R}$ such that

$$
y_{0}=\exp \left\{z-\int_{a}^{x} p(u) d u\right\}\left[z-\int_{a}^{x} \exp \left\{\int_{a}^{v} p(u) d u\right\}\left(k+\int_{a}^{v} \exp (\alpha x) \cos \beta x f(x) d x\right)\right]
$$

has the required properties, where $k=\left[\exp (\alpha a) \cos \beta a y^{\prime}(a)-(\exp (\alpha a) \cos \beta a)^{\prime} y(a)+\right.$ $c \exp (\alpha a) \cos \beta a y(a)]$.

2. Let $\alpha$ and $\beta$ be real constants. The following differential equation

$$
x^{2} y^{\prime \prime}+\alpha x y^{\prime}+\beta y+f(x)=0
$$

is called the Euler differential equation. It is exact when $\alpha-\beta=2$. By Theorem 2.1, it has the Hyers-Ulam stability.

In general, $\mu(x)$ is an integrating factor of Euler differential equation if it satisfies

$$
\left.x^{2} \mu(x)\right)^{\prime \prime}-\left(\alpha x \mu(x)^{\prime}+\beta \mu(x)=0 .\right.
$$

The Equation (14) can be written as

$$
x^{2} \mu^{\prime \prime}(x)+(4-\alpha) x \mu^{\prime}(x)+(2-\alpha+\beta) \mu(x)=0 .
$$

By the trial of $\mu(x)=x^{m}$, we show that

$$
m^{2}+(3-\alpha) m+(2-\alpha+\beta)=0 .
$$

From (15) we obtain

$$
m=\frac{-(3-\alpha) \pm \sqrt{(1-\alpha)^{2}-4 \beta}}{2} .
$$

Now we can use the above corollaries for the Hyers-Ulam stability of Euler differential equation. This result is comparable with [[9], Theorem 2] and the main results of [10].

3. Hermite's differential equation

$$
y^{\prime \prime}-2 x y^{\prime}+2 \lambda y+f(x)=0 \quad(\lambda \in \mathbb{R})
$$


is exact when $\lambda=-1$ and it has the Hyers-Ulam stability.

4. Chebyshev's differential equation

$$
\left(1-x^{2}\right) y^{\prime \prime}-x y^{\prime}+n^{2} y+f(x)=0 \quad(n \in \mathbb{Z})
$$

is exact when $n= \pm 1$. By Theorem 2.1, it has the Hyers-Ulam stability.

\title{
5. Legendre's differential equation
}

$$
\left(1-x^{2}\right) y^{\prime \prime}-2 x y^{\prime}+n(n+1) y+f(x)=0 \quad(n \in \mathbb{Z})
$$

is exact when $n(n+1)=0$ and it has the Hyers-Ulam stability.

\begin{abstract}
Author details
${ }^{1}$ Department of Mathematics Iran University of Science and Technology, Narmak, Tehran, Iran ${ }^{2}$ Department of Mathematics, Semnan University, P. O. Box 35195-363, Semnan, Iran ${ }^{3}$ Technical and Vocational University of Iran, Technical and Vocational Faculty of Tabriz, P. O. Box 51745-135, Tabriz, Iran ${ }^{4}$ Department of Mathematics, Research Institute for Natural Sciences, Hanyang University, Seoul 133-791, South Korea
\end{abstract}

\section{Authors' contributions}

All authors conceived of the study, participated in its design and coordination, drafted the manuscript, participated in the sequence alignment, and read and approved the final manuscript.

\section{Competing interests}

The authors declare that they have no competing interests.

Received: 1 December 2011 Accepted: 23 March 2012 Published: 23 March 2012

\section{References}

1. Ulam, SM: In Problems in Modern Mathematics, vol. VI,Science Ed., Wiley, New York (1940)

2. Hyers, DH: On the stability of the linear functional equation. Proc Natl Acad Sci. 27, 222-224 (1941)

3. Rassias, ThM: On the stability of the linear mapping in Banach spaces. Proc Am Math Soc. 72, 297-300 (1978)

4. Alsina, C, Ger, R: On some inequalities and stability results related to the exponential function. J Inequal Appl. 2, 373-380 (1998)

5. Takahasi, S-E, Miura, T, Miyajima, S: On the Hyers-Ulam stability of Banach space-valued differential equation $y^{\prime}=\lambda y$. Bull Korean Math Soc. 39, 309-315 (2002)

6. Jung, S: Hyers-Ulam stability of linear differential equations of first-order II. Appl Math Lett. 19, 854-858 (2006)

7. Ionascu, EJ: Ordinary Differential Equations-Lecture Notes. http://math.columbusstate.edu/ejionascu/papers/diffeqbook. pdf (2006)

8. Li, Y, Shen, Y: Hyers-Ulam stability of linear differential equations of second-order. Appl Math Lett. 23, 306-309 (2010)

9. Jung, S: Hyers-Ulam stability of linear differential equations of first-order III. J Math Anal Appl. 311, 139-146 (2005)

10. Jung, S, Min, S: On approximate Eular differential equations. Abstr Appl Anal 2009, 8 (2009). Art. ID 537963

doi:10.1186/1687-1847-2012-36

Cite this article as: Ghaemi et al: Hyers-ulam stability of exact second-order linear differential equations.

Advances in Difference Equations 2012 2012:36.

\section{Submit your manuscript to a SpringerOpen ${ }^{\odot}$ journal and benefit from:}

- Convenient online submission

- Rigorous peer review

- Immediate publication on acceptance

- Open access: articles freely available online

- High visibility within the field

- Retaining the copyright to your article

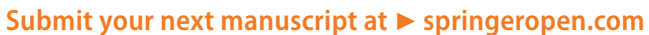

